\title{
Comparaison des besoins en soins de santé des personnes vivant avec, et sans, le VIH dans le contexte des soins à domicile et des soins de longue durée au Canada
}

\author{
Foebel $A D^{1 *}$, Hirdes JP ${ }^{1}$, Boodram $C^{2}$, Lemick $\mathrm{R}^{3}$, Tai JW³ ${ }^{3}$, Comeau $\mathrm{RL}^{2}$
}

\section{Résumé}

Contexte : Depuis l'introduction des traitements antirétroviraux hautement actifs (HAART), I'infection au VIH est devenue une infection chronique qui peut être prise en charge, et les personnes qui en sont atteintes vivent maintenant plus longtemps. Il faut donc s'attendre à ce que des personnes âgées vivant avec le VIH commencent à demander des services dans l'ensemble du continuum des soins de santé. Cependant, on ne sait pas si les besoins de ces personnes en matière de soins diffèrent de ceux des personnes qui ne sont pas porteuses du $\mathrm{VIH}$.

Objectifs : Comparer les caractéristiques démographiques, les affections chroniques, les infections et les problèmes de santé mentale chez les personnes séropositives et séronégatives pour le $\mathrm{VIH}$ dans les milieux des soins à domicile, des soins de longue durée et des soins continus complexes au Canada.

Méthodologie : Cette étude transversale a utilisé les données interRAl pour comparer les caractéristiques des personnes séropositives et séronégatives dans les milieux des soins de longue durée, des soins continus complexes et des soins à domicile. Des analyses du chi carré ont examiné les différences entre les groupes sur le plan des co-infections, des maladies chroniques et des problèmes de santé mentale.

Résultats : Les données de 1200073 personnes, dont 1608 (0,13\%) étaient séropositives, ont été analysées. Dans l'ensemble, les personnes séropositives ont présenté davantage de co-infections, mais moins de maladies chroniques, que les personnes séronégatives. La dépression, l'isolement social et l'usage de médicaments psychotropes ont généralement été plus fréquents dans la cohorte des personnes séropositives.

Conclusion : Les personnes vivant avec le VIH forment une petite cohorte de personnes qui ont des besoins complexes en ce qui a trait aux soins à domicile ou en établissements, et ces besoins diffèrent de ceux des personnes qui ne sont pas porteuses du VIH. Une meilleure compréhension du contexte dans lequel ces besoins surviennent à mesure que les personnes séropositives vieillissent nous aidera à proposer de meilleures interventions.

\section{Affiliations}

'School of Public Health and Health Systems, Université de Waterloo, Waterloo (Ontario)

${ }^{2}$ Centre de la lutte contre les maladies transmissibles et les infections, Agence de la santé publique du Canada, Ottawa (Ontario)

${ }^{3}$ Région de l'Ontario, Agence de la santé publique du Canada, Toronto (Ontario)

*Correspondance : adfoebel@ uwaterloo.ca

Citation proposée : Foebel AD, Hirdes JP, Boodram C, Lemick R, Tai JW, Comeau RL. Comparaison des besoins en soins de santé des personnes vivant avec, et sans, le VIH dans le contexte des soins à domicile et des soins de longue durée au Canada. Relevé des maladies transmissibles au Canada 2016;42:59-64.

https://doi.org/10.14745/ccdr.v42i03a01f

\section{Introduction}

Dans bon nombre de pays développés, l'infection au VIH est devenue une maladie chronique, grâce aux progrès réalisés dans la prise en charge de cette infection, notamment depuis I'introduction du traitement antirétroviral hautement actif (HAART). Nous observons des changements dans les profils de comorbidité des personnes vivant avec le $\mathrm{VIH} /$ sida, ces profils passant de maladies définissant le sida à des maladies associées au VIH mais ne définissant pas le sida, comme les maladies cardiovasculaires et les néphropathies $(1,2)$. De plus, près des deux tiers des personnes âgées vivant avec le

$\mathrm{VIH} /$ sida sont aujourd'hui atteintes d'autres affections chroniques (3-11). Ces affections chroniques apparaissent plus tôt que chez les personnes séronégatives $(1,5,11)$ et les comorbidités incluent davantage d'infections dues à une immunosuppression et aux traitements antirétroviraux hautement actifs $(1,2,12)$. Enfin, malgré les progrès réalisés dans le domaine des HAART 
traitements antirétroviraux hautement actifs, les troubles neurocognitifs associés au VIH sont des effets à long terme bien connus de cette infection (13). Il est probable que les personnes âgées vivant avec le $\mathrm{VIH} /$ sida diffèrent considérablement des personnes plus jeunes qui en sont atteintes, ainsi que des adultes âgés non porteurs du $\mathrm{VIH}$; les besoins en matière de soins des personnes âgées vivant avec le $\mathrm{VIH} /$ sida sont donc uniques.

On s'attend à ce que la prévalence du VIH au Canada suive les projections aux États-Unis où l'on estime que la moitié des personnes séropositives seront âgées de 50 ans et plus d'ici 2050 (14). Ce changement démographique aura d'importantes répercussions en ce qui a trait à la prise en charge de la maladie et au milieu de soins que choisiront ces personnes. II est probable qu'un grand nombre d'adultes porteurs du VIH finiront par avoir besoin de services de soutien, de traitement et de soins dans des établissements de soins non actifs, par exemple à domicile ou en établissements de soins de longue durée (15). On ignore si les fournisseurs de ces services sont prêts à répondre aux besoins uniques de ces personnes en matière de soins, mais on sait que les pressions qui s'exerceront sur ces systèmes augmenteront à mesure que les personnes séropositives chercheront de plus en plus à obtenir des soins.

Les données d'évaluation normalisées d'interRAl peuvent aider à déterminer si ces besoins sont satisfaits dans les différents milieux de soins de santé au Canada. interRAl est un consortium international de recherche sans but lucratif, qui a été mis sur pied au départ par des cliniciens et des chercheurs dans le but d'améliorer la qualité des soins et la qualité de vie dans les maisons de soins infirmiers aux États-Unis

(www.interRAl.org) $(16,17)$. Les instruments d'évaluation d'interRAl ont été conçus de manière à proposer une approche intégrée commune pour l'évaluation normalisée des populations vulnérables qui requièrent des soins complexes, notamment les personnes nécessitant des soins à domicile ou vivant en maisons de soins infirmiers $(16,18-20)$. Les instruments d'évaluation MDS 2.0 (ensemble minimal de données, version 2.0) et RAI-HC (soins à domicile) ont été validés pour l'analyse des milieux des soins à domicile et des soins de longue durée, respectivement (19-21). Bien que ces instruments soient utilisés au Canada depuis 1996 et 2002, respectivement, on ne les a jamais utilisés pour suivre les besoins en soins de santé des personnes séropositives.

Nous possédons à l'heure actuelle peu de données sur les besoins des personnes séropositives en termes de soins à domicile ou en établissements. La présente étude avait pour but de comparer les besoins des personnes vivant avec, et sans, le $\mathrm{VIH}$ dans les milieux des soins à domicile et des soins de longue durée au Canada, en s'intéressant plus particulièrement aux co-infections, aux comorbidités et aux problèmes de santé mentale.

\section{Méthodologie}

\section{Milieux}

Dans cette étude, on a examiné des données portant sur les milieux des soins à domicile (SD), des soins de longue durée
(SLD) et des soins continus complexes (SCC) au Canada, lesquels sont définis dans l'encadré qui suit.

\section{Définition des milieux de soins}

Soins à domicile (SD) : Services qui incluent un ensemble de soins de soutien à la personne, de soins infirmiers et de soins de réadaptation, qui sont dispensés au domicile du client. Remarque : On s'attend à ce que les patients de longue durée aient besoin de ces services pendant 60 jours et plus.

Soins de longue durée (SLD) : Maisons de soins infirmiers privées, publiques ou caritatives, qui offrent des soins réglementés aux personnes dont l'état de santé est stable mais qui requièrent des soins 24 heures par jour.

Soins continus complexes (SCC) : Hôpitaux ou services dispensant des soins en phase postaiguë aux personnes qui présentent des atteintes plus graves ou des problèmes de santé plus complexes, ou dont les besoins en santé mentale dépassent généralement ceux pouvant être pris en charge dans les maisons de soins infirmiers. Remarque : Ces établissements n'existent qu'en Ontario et au Manitoba.

L'information sur ces trois milieux de soins a été compilée à partir des données obtenues à l'aide de deux instruments d'évaluation canadiens d'interRAl. Les données pour l'évaluation du milieu des soins à domicile ont été recueillies à l'aide de I'instrument RAI-HC, alors que les données pour les échantillons liés aux soins en établissements (SLD et SCC) ont été obtenues à I'aide de l'instrument interRAI MDS 2.0. Les données aux fins de ces évaluations ont été extraites de deux dépôts nationaux de données gérés par I'Institut canadien d'information sur la santé (ICIS), soit le Système d'information sur les services à domicile (SISD) et le Système d'information sur les soins de longue durée (SISLD) $(22,23)$. L'accès à ces données aux fins de la recherche a été rendu possible grâce à des accords de partage des données conclus entre I'ICIS, interRAI et I'Université de Waterloo. Les données pour l'évaluation RAI-HC ont été recueillies auprès de tous les patients ayant obtenu des services à domicile prolongés de 2002 à 2014. Pour les populations recevant des soins de longue durée et des soins continus complexes, les évaluations MDS 2.0 ont porté sur des données recueillies entre 1996 et 2014.

\section{Population}

L'étude a porté sur toutes les personnes dans ces milieux de soins qui avaient fait l'objet d'une évaluation interRAl. Les évaluations RAI-HC faites en milieux hospitaliers ont été exclues, car ces évaluations servent à déterminer l'admissibilité aux soins de longue durée et non à la planification des soins à domicile.

L'échantillon sur le milieu des soins à domicile était composé de personnes de la Colombie-Britannique, du Manitoba, de I'Ontario, de la Nouvelle-Écosse et du Yukon. L'échantillon sur les soins de longue durée incluait des personnes de l'Alberta, de la Colombie-Britannique, du Manitoba, du Nouveau-Brunswick, de Terre-Neuve-et-Labrador, de la Nouvelle-Écosse, de l'Ontario, de la Saskatchewan et du Yukon. Les données sur les soins continus complexes étaient disponibles pour l'Ontario et le Manitoba. 


\section{Mesures}

Les instruments d'interRAl comprennent un ensemble exhaustif d'éléments de base qui décrivent les caractéristiques démographiques et cliniques du client et qui aident à la planification des soins. Les renseignements démographiques au sujet de l'âge, du sexe et de l'état matrimonial du sujet (" marié » faisant référence aux personnes mariées et aux conjoints de fait, y compris les partenaires de même sexe) peuvent être obtenus de tous les dossiers d'interRAl.

Les mesures utilisées pour cette étude incluaient notamment des affections chroniques souvent associées au vieillissement, ainsi que des affections auxquelles les personnes vivant avec le VIH/sida pourraient davantage être exposées (notamment des infections et des comorbidités liées à la santé mentale). La plupart des données étaient disponibles à la fois pour le $\mathrm{RAI}-\mathrm{HC}$ et le MDS 2.0. Dans ces deux instruments, les listes de vérification des diagnostics étaient disponibles pour toutes les maladies chroniques (insuffisance cardiaque, emphysème/ maladie pulmonaire obstructive chronique [MPOC], diabète, cancer, tout trouble psychiatrique et autres maladies cardiovasculaires) et maladies infectieuses (pneumonie, tuberculose et infections des voies urinaires [UVI]) évaluées dans cette étude. Cependant, les diagnostics de certaines maladies infectieuses (infections antibiorésistantes, cellulite, infection à Clostridium difficile, conjonctivite, infections des voies respiratoires, septicémie, infections transmissibles sexuellement [ITS] et hépatite virale) n'étaient disponibles qu'à partir des listes de vérification de l'instrument MDS 2.0 et n'ont pu être mesurés dans l'échantillon sur les soins à domicile.

Dans le cas des symptômes de santé mentale (notamment I'anxiété, les comportements agressifs, les hallucinations et l'isolement social), des éléments précis servent à indiquer la présence ou l'absence du symptôme dans les deux instruments (RAI-HC et MDS 2.0). Les symptômes de dépression ont été évalués selon l'échelle de dépression intégrée dans les deux instruments d'évaluation interRAl, un score de trois ou plus selon cette échelle indiquant une dépression possible (24). Les instruments prennent également en compte l'usage de médicaments psychotropes (antipsychotiques, antidépresseurs, anxiolytiques et sédatifs) au cours des sept derniers jours.

\section{Analyse}

Les différences entre les caractéristiques des personnes séropositives et séronégatives dans chaque milieu de soins ont été analysées à l'aide du test du chi carré (niveau de signification $p<0,05)$ pour toutes les variables d'intérêt. L'âge a été réparti en six catégories afin de pouvoir distinguer les sujets très jeunes et très vieux. Toutes les analyses ont été réalisées à l'aide du système SAS, version 9.4 (SAS Institute Inc., Cary, Caroline du Nord).

\section{Résultats}

L'échantillon total était composé de 1200073 personnes (tableau 1). La prévalence du VIH était de 0,13\%, ce qui est légèrement inférieur au pourcentage dans l'ensemble de la population canadienne $(0,21 \%$ en 2014$)(25)$; une forte proportion des sujets de l'échantillon provenaient de l'Ontario (76\%). L'échantillon des SLD était composé de 356621 sujets, au sein desquels la prévalence globale du VIH était de 0,05 \%; dans cinq régions (Nouveau-Brunswick, Terre-Neuve-et-Labrador, Nouvelle-Écosse, Saskatchewan et Yukon); toutefois, aucun cas de VIH n'a été recensé. Le taux de prévalence du VIH parmi les sujets nécessitant des soins continus complexes a été beaucoup plus élevé, s'établissant à $0,19 \%$. Enfin, la prévalence globale du $\mathrm{VIH}$ dans le large échantillon des sujets recevant des soins à domicile $(n=618301)$ a été de $0,16 \%$, variant de $0,07 \%$ en Nouvelle-Écosse à $0,49 \%$ en Colombie-Britannique.

Tableau 1 : Résumé de l'échantillon de l'étude selon le milieu de soins, Canada ( $n=1200$ 073)

\begin{tabular}{|c|c|c|c|}
\hline Milieu de soins & VIH/sida & Total & $\begin{array}{l}\text { Prévalence du VIH } \\
\text { (\%) }\end{array}$ \\
\hline Soins de longue durée ${ }^{1}$ & 178 & 356621 & 0,05 \\
\hline Soins continus complexes ${ }^{2}$ & 423 & 225151 & 0,19 \\
\hline Soins à domicile ${ }^{3}$ & 1007 & 618301 & 0,16 \\
\hline TOTAL & 1608 & 1200073 & 0,13 \\
\hline \multicolumn{4}{|c|}{$\begin{array}{l}\text { Abréviations : sida : syndrome d'immunodéficience acquise; VIH : virus de l'immunodéficience humaine. } \\
\text { 'Échantillon constitué de sujets de l'Alberta }(10 \%) \text {, de la Colombie-Britannique }(17 \%) \text {, } \\
\text { du Manitoba }(4 \%) \text {, du Nouveau-Brunswick }(<1 \%) \text {, de Terre-Neuve-et-Labrador }(<1 \%) \text {, } \\
\text { de la Nouvelle-Écosse }(<1 \%) \text {, de l'Ontario }(58 \% \text {, de la Saskatchewan }(9 \%) \text { et du Yukon }(<1 \%) \text {. } \\
\text { 'Échantillon constitué de sujets du Manitoba }(0,3 \%) \text { et de l'Ontario }(99,7 \%) \text {. }\end{array}$} \\
\hline \multicolumn{4}{|c|}{$\begin{array}{l}\text { 3échantillon constitué de sujets de la Colombie-Britannique }(11 \%) \text {, du Manitoba }(2 \%) \text {, } \\
\text { de la Nouvelle-Écosse }(8 \%) \text {, de I'Ontario }(78 \%) \text { et du Yukon }(<1 \%) \text {. }\end{array}$} \\
\hline
\end{tabular}

Le tableau 2 présente les caractéristiques démographiques et les caractéristiques diagnostiques de l'échantillon. Dans les trois milieux de soins, des différences quant à certaines caractéristiques clés ont été observées entre les groupes séropositifs et séronégatifs. Environ $60 \%$ à $65 \%$ des sujets séronégatifs dans les trois milieux étaient des femmes, comparativement à une proportion de $20 \%$ à $30 \%$ parmi les sujets séropositifs. Entre $95 \%$ et $99 \%$ des personnes séronégatives étaient âgées de plus de 50 ans, comparativement à une proportion de $47 \%$ à $72 \%$ parmi les sujets séropositifs. Les taux de mariage dans les groupes séropositifs étaient deux fois moins élevés que dans les groupes séronégatifs, les hommes étant moins susceptibles que les femmes d'être mariés, sauf dans le groupe des soins à domicile où la proportion de sujets mariés a été moins élevée, autant chez les hommes que chez les femmes séropositifs.

\section{Co-infections}

Les diagnostics de maladie ont varié selon le milieu de soins (tableau 2). Dans le cas des maladies infectieuses, des taux variables ont été observés; la prévalence de la pneumonie a toutefois été plus élevée chez les personnes séropositives dans les milieux des soins continus complexes et des soins à domicile. Dans tous les milieux de soins, les taux de tuberculose ont été plus élevés dans les cohortes séropositives. Les taux d'infections antibiorésistantes, de cellulite et d'infection à $C$. difficile ont tous été plus élevés dans la cohorte séropositive dans les milieux de soins continus complexes et de soins de longue durée. Dans le groupe des soins complexes continus, la prévalence de la septicémie a été plus élevée chez les personnes séropositives, alors que les infections transmissibles sexuellement ont été beaucoup plus fréquentes dans les cohortes séropositives des 
Tableau 2 : Caractéristiques démographiques, co-infections, diagnostics de comorbidités et problèmes de santé mentale de l'échantillon canadien, selon le milieu de soins ( $n=1200$ 073)

\begin{tabular}{|c|c|c|c|c|c|c|c|c|c|}
\hline \multirow[b]{2}{*}{ Caractéristiques } & \multicolumn{3}{|c|}{ Soins de longue durée (\%) } & \multicolumn{3}{|c|}{ Soins continus complexes (\%) } & \multicolumn{3}{|c|}{ Soins à domicile (\%) } \\
\hline & $\begin{array}{c}\text { Non } \\
\text { porteurs du VIH } \\
(\mathrm{n}=356443)\end{array}$ & $\begin{array}{l}\text { VIH } \\
178\end{array}$ & Valeur $p$ & $\begin{array}{c}\text { Non porteurs } \\
\text { du VIH } \\
224728\end{array}$ & $\begin{array}{l}\text { VIH } \\
423\end{array}$ & Valeur $p$ & $\begin{array}{c}\text { Non } \\
\text { porteurs du VIH } \\
617294\end{array}$ & $\begin{array}{c}\text { VIH } \\
1007\end{array}$ & Valeur $p$ \\
\hline Âge (ans) & & & $<0,0001$ & & & $<0,0001$ & & & $<0,0001$ \\
\hline 0 à 39 & 0,3 & 5,6 & & 1,3 & 20,8 & & 2,2 & 11,1 & \\
\hline 40 à 49 & 0,6 & 18,5 & & 2,3 & 31,9 & & 3,0 & 29,6 & \\
\hline 50 à 64 & 4,2 & 43,3 & & 10,5 & 28,1 & & 11,6 & 37,5 & \\
\hline 65 à 74 & 8,5 & 18,5 & & 18,0 & 9,5 & & 14,8 & 11,3 & \\
\hline 75 à 84 & 28,0 & 10,7 & & 36,7 & 5,7 & & 33,0 & 6,3 & \\
\hline 85 et plus & 58,4 & ND & & 31,4 & 4,0 & & 35,4 & 4,2 & \\
\hline Femmes & 65,8 & 22,5 & $<0,0001$ & 57,6 & 26,5 & $<0,0001$ & 62,8 & 30,3 & $<0,0001$ \\
\hline Mariés & 23,0 & 10,7 & $<0,0001$ & 39,2 & 18,0 & $<0,0001$ & 38,1 & 12,4 & $<0,0001$ \\
\hline Hommes & 39,3 & 9,4 & $<0,0001$ & 55,5 & 17,0 & $<0,0001$ & 56,5 & 11,6 & $<0,0001$ \\
\hline Femmes & 14,5 & 15,0 & 0,92 & 27,2 & 20,5 & 0,11 & 27,6 & 14,5 & $<0,0001$ \\
\hline \multicolumn{10}{|l|}{ Infections } \\
\hline Pneumonie & 2,6 & 3,9 & 0,28 & 7,3 & 13,2 & $<0,0001$ & 2,9 & 9,8 & $<0,0001$ \\
\hline Tuberculose & 0,1 & ND & ND & 0,1 & 2,6 & $<0,0001$ & 0,1 & 4,1 & $<0,0001$ \\
\hline IVU & 7,0 & 6,7 & 0,88 & 16,5 & 9,5 & $<0,0001$ & 4,8 & 6,1 & 0,05 \\
\hline Résistance aux antibiotiques & 4,0 & 12,4 & $<0,0001$ & 6,7 & 9,9 & 0,007 & S.O. & S.O. & S.O. \\
\hline Cellulite & 1,0 & ND & ND & 1,5 & 3,6 & 0,0005 & S.O. & S.O. & S.O. \\
\hline Clostridium difficile & 0,6 & ND & ND & 2,0 & 5,0 & $<0,0001$ & S.O. & S.O. & S.O. \\
\hline Conjonctivite & 0,5 & 0 & 0,34 & 0,6 & 1,9 & 0,0003 & S.O. & S.O. & S.O. \\
\hline Infection respiratoire & 2,7 & ND & ND & 3,6 & 4,5 & 0,3 & S.O. & S.O. & S.O. \\
\hline Septicémie & 0,6 & ND & ND & 1,4 & 4,5 & $<0,0001$ & S.O. & S.O. & S.O. \\
\hline ITS & 0,1 & 6,7 & $<0,0001$ & 0,1 & 12,1 & $<0,0001$ & S.O. & S.O. & S.O. \\
\hline Hépatite virale & 1,2 & 23,6 & $<0,0001$ & 0,5 & 18,7 & $<0,0001$ & S.O. & S.O. & S.O. \\
\hline \multicolumn{10}{|l|}{ Diagnostic } \\
\hline Insuffisance cardiaque & 15,4 & 6,4 & 0,001 & 14,1 & 4,7 & $<0,0001$ & 13,0 & 6,1 & $<0,0001$ \\
\hline Emphysème/MPOC & 17,1 & 18,6 & 0,61 & 18,3 & 14,7 & 0,05 & 17,7 & 19,8 & 0,09 \\
\hline Diabète & 24,7 & 24,2 & 0,87 & 25,5 & 12,4 & $<0,0001$ & 25,2 & 15,6 & $<0,0001$ \\
\hline Cancer & 10,9 & 8,1 & 0,25 & 27,5 & 17,5 & $<0,0001$ & 17,7 & 12,5 & $<0,0001$ \\
\hline Tout diagnostic psychiatrique & 53,4 & 52,8 & 0,87 & 40,6 & 48,0 & 0,002 & 30,0 & 46,2 & $<0,0001$ \\
\hline Autres maladies cardiovasculaires & 66,1 & 32,6 & $<0,0001$ & 60,4 & 27,2 & $<0,0001$ & 67,4 & 32,2 & $<0,0001$ \\
\hline \multicolumn{10}{|l|}{ Santé mentale } \\
\hline Symptômes d'anxiété & 32,8 & 30,3 & 0,49 & 24,7 & 29,1 & 0,04 & 17,7 & 21,1 & 0,006 \\
\hline Troubles anxieux & 8,0 & 7,0 & 0,63 & 7,0 & 8,8 & 0,15 & S.O. & S.O. & S.O. \\
\hline Dépression ${ }^{1}$ & 31,1 & 23,9 & 0,04 & 22,7 & 25,9 & 0,0008 & 18,1 & 25,7 & 0,004 \\
\hline Tout comportement agressif & 43,9 & 48,3 & 0,23 & 27,1 & 33,6 & 0,003 & 9,9 & 7,9 & 0,03 \\
\hline Hallucinations/délire & 5,9 & ND & 0,44 & 7,2 & 7,6 & 0,75 & 4,3 & 3,3 & 0,12 \\
\hline Isolement social & 4,9 & 9,1 & 0,09 & 6,3 & 13,4 & $<0,0001$ & 15,4 & 19,3 & 0,0006 \\
\hline \multicolumn{10}{|l|}{ Utilisation de psychotropes } \\
\hline Antipsychotiques & 30,9 & 42,7 & 0,0006 & 19,7 & 27,0 & 0,0002 & 10,7 & 20,0 & $<0,0001$ \\
\hline Antidépresseurs & 46,8 & 58,4 & 0,002 & 29,1 & 33,6 & 0,04 & 24,9 & 36,3 & $<0,0001$ \\
\hline Anxiolytiques & 14,7 & 22,5 & 0,003 & 29,8 & 39,5 & $<0,0001$ & 16,4 & 22,2 & $<0,0001$ \\
\hline Sédatifs & 11,5 & 23,0 & $<0,0001$ & 15,5 & 15,6 & 0,94 & 20,9 & 25,2 & 0,0008 \\
\hline
\end{tabular}

Abréviations : MPOC : maladie pulmonaire obstructive chronique; VIH : virus de l'immunodéficience humaine; ITS : infection transmissible sexuellement; IVU : infection des voies urinaires;

ND : non déclaré (en raison de la petite taille des cellules; $<9$ cas).

'Les symptômes de dépression probable étaient définis comme un score $\geq 3$ selon l'échelle de dépression.

groupes de soins de longue durée $(6,7 \% \mathrm{p} / \mathrm{r}$ à $0,1 \%)$ et de soins complexes continus $(12,1 \% \mathrm{p} / \mathrm{r}$ à $0,1 \%)$.

\section{Diagnostics de maladies chroniques}

La prévalence des maladies chroniques a été dans l'ensemble moins élevée chez les personnes vivant avec le $\mathrm{VIH} / \mathrm{sida}$, à l'exception des troubles psychiatriques chroniques qui ont été plus répandus chez les personnes séropositives recevant des soins à domicile $(p<0,0001)$ (tableau 2). Dans le milieu des soins de longue durée, le taux d'insuffisance cardiaque a été plus faible chez les personnes séropositives, alors que les taux de MPOC, de diabète et de cancer ont été comparables dans les groupes séropositifs et séronégatifs. Dans les milieux des soins continus complexes et des soins à domicile, les personnes séropositives ont affiché des taux de MPOC comparables à ceux observés chez les personnes séronégatives, mais leurs taux d'insuffisance cardiaque, de diabète et de cancer ont été moins élevés. Les autres maladies cardiovasculaires ont été nettement moins fréquentes chez les personnes séropositives dans les trois milieux de soins $(p<0,0001)$.

\section{Troubles de santé mentale}

Dans l'ensemble, les troubles de santé mentale ont été répandus et comparables chez les résidents des établissements de soins de longue durée, qu'ils aient ou non été porteurs du VIH (tableau 2). Dans les milieux des soins continus complexes et des soins à domicile, les taux de symptômes de l'anxiété ont été légèrement plus élevés chez les personnes vivant avec le $\mathrm{VIH} /$ sida, mais les différences n'étaient pas statistiquement significatives. Alors que les comportements agressifs étaient plus répandus chez les personnes séropositives dans le milieu des soins continus complexes $(33,6 \% \mathrm{p} / \mathrm{r}$ à $27,1 \%)$, ils ont été moins fréquents chez les personnes séropositives recevant des soins à domicile $(7,9 \% \mathrm{p} / \mathrm{r}$ à 9,9\%). L'isolement social a été nettement plus marqué chez les personnes séropositives que chez les personnes séronégatives dans les milieux des soins continus complexes et des soins à domicile $(p<0,0001)$. Enfin, dans tous les milieux de soins, les taux d'utilisation de médicaments psychotropes ont été plus élevés chez les personnes séropositives que chez les personnes séronégatives, mais aucune différence statistiquement significative n'a été observée dans I'utilisation des antidépresseurs et des sédatifs dans le milieu des soins continus complexes. 


\section{Discussion}

Il s'agit de la plus vaste étude menée à ce jour auprès de personnes vivant avec le $\mathrm{VIH} /$ sida dans différents milieux de soins. Cette étude, qui a porté sur 1608 personnes vivant avec le VIH/sida dans l'ensemble du Canada, est venue confirmer les résultats d'une étude antérieure menée auprès d'une cohorte ontarienne (26). Par comparaison à leurs homologues non infectés par le $\mathrm{VIH}$, les personnes âgées vivant avec le $\mathrm{VIH} /$ sida ont présenté davantage de co-infections, moins de maladies chroniques et un profil de santé mentale comparable, bien qu'elles aient eu tendance à consommer plus de médicaments psychotropes. À l'exception des infections urinaires, les co-infections ont été plus répandues chez les personnes vivant avec le $\mathrm{VIH} /$ sida dans les trois milieux de soins, ce qui est compatible avec les résultats de recherches antérieures $(27,28)$. Les taux de comorbidités variaient chez les personnes vivant avec le $\mathrm{VIH} /$ sida, la prévalence globale étant toutefois inférieure aux taux déclarés lors d'études antérieures (3-11). Ces résultats pourraient être attribuables au jeune âge des personnes vivant avec le VIH/sida dans cette étude, mais il nous a été impossible de le confirmer car la taille de l'échantillon des personnes vivant avec le $\mathrm{VIH} /$ sida n'était pas suffisante pour effectuer une stratification selon l'âge.

Cette étude confirme également la forte prévalence des diagnostics psychiatriques, de la dépression et des symptômes de l'anxiété chez les personnes âgées vivant avec le $\mathrm{VIH} /$ sida $(2,7,8,11,29-31)$. II convient toutefois de souligner que la prévalence des troubles anxieux et des hallucinations ou du délire a également été assez élevée chez les personnes séronégatives dans ces milieux de soins. La prévalence de la dépression a atteint près de $25 \%$ chez les personnes vivant avec le $\mathrm{VIH} /$ sida, ce qui est légèrement supérieur au taux de $21 \%$ observé dans les maisons de soins infirmiers des États-Unis $(28,32)$.

Les taux d'utilisation de médicaments psychotropes, nettement plus élevés chez les personnes séropositives, semblent être une nouvelle observation. Cependant, comme il s'agit d'un ensemble de données descriptives, on ne peut établir la raison expliquant ce résultat. Les taux d'isolement social ont été plus élevés chez les personnes vivant avec le $\mathrm{VIH} /$ sida, en particulier dans le milieu des soins à domicile. L'isolement social, reconnu pour être fréquent chez les personnes âgées vivant avec le $\mathrm{VIH} /$ sida, augmente avec l'âge (3) et peut mener à la solitude et à la dépression (2).

Les principales forces de cette étude tiennent à l'inclusion de vastes échantillons provenant de multiples sites, ainsi que de données portant sur différentes régions du Canada. Ces résultats peuvent être considérés comme représentatifs des trois milieux de soins examinés, car les données interRAl sont complètes là où elles sont obligatoires. Enfin, ces données ont permis de caractériser les besoins précis des personnes âgées vivant avec le $\mathrm{VIH} /$ sida alors que celles-ci commencent à explorer les options de soins qui s'offrent à mesure qu'elles vieillissent.

La surreprésentation des données de l'Ontario et la variabilité du taux d'adoption des instruments interRAl selon la région du Canada constituent toutefois d'importantes limitations. De plus, ces résultats ne peuvent être comparés à ceux portant sur des personnes vivant avec le $\mathrm{VIH} /$ sida qui n'ont pas accès à ces soins ou qui ont accès à des soins propres au VIH/sida dans d'autres milieux. Enfin, cette étude transversale n'a pu établir de relations de cause à effet.

Avec le vieillissement de la population, la demande de soins de longue durée pour soutenir, traiter et prendre en charge des personnes âgées vivant avec le $\mathrm{VIH} /$ sida augmentera. Le défaut de prendre en compte les besoins uniques de ce groupe en matière de soins risque d'aggraver les résultats sur la santé et d'accroître les pressions sur les systèmes de santé (33). II pourrait être judicieux d'examiner plus à fond la prévalence plus élevée de l'utilisation des médicaments psychotropes chez les personnes séropositives, notamment dans le contexte du débat actuel concernant la pertinence de ces traitements en général $(34,35)$. Il s'impose de surveiller ces tendances.

D'autres recherches portant sur un échantillon national étendu de personnes âgées vivant avec le $\mathrm{VIH} /$ sida fourniraient des informations sur les régions qui ont été exclues de la présente étude. L'examen des options stratégiques visant à améliorer les soins et l'accès aux soins dans ces milieux devrait également être une priorité, en particulier dans le contexte des soins à domicile où l'amélioration des services pourrait retarder ou prévenir les hospitalisations et les admissions en établissements de soins de longue durée.

\section{Conclusions}

Davantage de personnes vivant avec le $\mathrm{VIH} /$ sida vivent aujourd'hui plus longtemps et doivent composer avec la réalité du VIH et du vieillissement. Nos conclusions portent à croire que les personnes vivant avec le $\mathrm{VIH} /$ sida dans les milieux des soins à domicile, des soins de longue durée et des soins continus complexes ont d'importants besoins en ce qui a trait à la prise en charge des co-infections, des comorbidités et des troubles de santé mentale, besoins qui les distinguent de leurs homologues non porteurs du VIH.

\section{Financement}

A.D. Foebel, Ph. D. et J.P. Hirdes, Ph. D., ont reçu des fonds de l'Agence de la santé publique du Canada [contrat $n^{\circ} 4500326437$ ] pour mener un large projet de recherche sur lequel le présent article est fondé.

\section{Conflit d'intérêts}

Tous les auteurs ont confirmé n'avoir aucun conflit d'intérêts.

\section{Références}

1. Deeks SG, Lewin SR, Havlir DV. The end of AIDS: HIV infection as a chronic disease. Lancet. 2013;382(9903):1525-33.

2. High KP, Brennan-Ing M, Clifford DB, Cohen MH, Currier J, Deeks SG, et al. HIV and aging: state of knowledge and areas of critical need for research. A report to the NIH Office of AIDS Research by the HIV and Aging Working Group. J Acquir Immune Defic Syndr. 2012;60 Suppl 1:S1-18. 
3. Greysen SR, Horwitz LI, Covinsky KE, Gordon K, Ohl ME, Justice AC. Does social isolation predict hospitalization and mortality among HIV+ and uninfected older veterans? J Am Geriatr Soc. 2013;61(9):1456-63.

4. Justice AC. HIV and aging: time for a new paradigm. Curr HIV/AIDS Reports. 2010;7(2):69-76.

5. Onen NF, Overton ET, Seyfried W, Stumm ER, Snell M, Mondy $\mathrm{K}$, et al. Aging and HIV infection: a comparison between older HIV-infected persons and the general population. HIV Clin Trials. 2010;11(2):100-9.

6. Rodriguez-Penney AT, ludicello JE, Riggs PK, Doyle K, Ellis RJ, Letendre SL, et al. Co-morbidities in persons infected with HIV: increased burden with older age and negative effects on health-related quality of life. AIDS Patient Care STDs. 2013;27(1): 5-16.

7. Balderson BH, Grothaus L, Harrison RG, McCoy K, Mahoney C, Catz S. Chronic illness burden and quality of life in an aging HIV population. AIDS Care. 2013;25(4):451-8.

8. Ball SC. The aging HIV population. Clin Pract. 2014;11(2):221-321.

9. Brennan-Ing M, Seidel L, London AS, Cahill S, Karpiak SE. Service utilization among older adults with HIV: the joint association of sexual identity and gender. J Homosex. 2014;61(1):166-96.

10. Cardoso SW, Torres TS, Santini-Oliveira M, Marins LM, Veloso VG, Grinsztejn B. Aging with HIV: a practical review. Braz J Infect Dis. 2013;17(4):464-79.

11. Havlik RJ, Brennan M, Karpiak SE. Comorbidities and depression in older adults with HIV. Sex Health. 2011;8(4):551-9.

12. Deeks SG, Tracy R, Douek DC. Systemic effects of inflammation on health during chronic HIV infection. Immunity. 2013;39(4):633-45.

13. Ibirt D, Mahlab-Guri K, Bezalel-Rosenberg S, Gill H, Attali M, Asher I. HIV-associated neurocognitive disorders (HAND). Israel Med Assoc J. 2015;17(1):54-9.

14. Cahill S, Darnell B, Guidry JA, Krivo-Kaufman A, Schaefer N, Urbano $L$, et al. Growing older with the epidemic: HIV and aging. New York: Gay Men's Health Crisis; 2010 http://www.hivandrehab.ca/wpcontent/uploads/a_pa_aging10_emb2.pdf15.

15. Wallach I, Brotman S. Ageing with HIV/AIDS: a scoping study among people aged 50 and over living in Quebec. Ageing Soc. 2013;33:1212-42.

16. Bernabei R, Gray L, Hirdes J, Pei X, Henrard JC, Jonsson PV, et al. International gerontology. In: High K, Halter J, Asthana S, Ouslander J, Tinetti M, Studenski S, Hazzard W, editors. Hazzard's Geriatric Medicine and Gerontology. 6th ed. New York: McGraw Medical; 2009. p. 69-96.

17. Morris JN, Hawes C, Fries BE, Phillips CD, Mor V, Katz S, et al. Designing the national resident assessment instrument for nursing homes. Gerontologist. 1990;30(3):293-307.

18. Hirdes JP, Fries BE, Morris JN, Steel K, Mor V, Frijters D, et al. Integrated health information systems based on the RAI/MDS series of instruments. Healthc Manage Forum. 1999;12(4):30-40.

19. Carpenter GI. Accuracy, validity and reliability in assessment and in evaluation of services for older people: the role of the interRAI MDS assessment system. Age Ageing. 2006;35(4):327-9.
20. Hirdes JP, Ljunggren G, Morris JN, Frijters DH, Finne Soveri H, Gray $L$, et al. Reliability of the interRAl suite of assessment instruments: a 12 -country study of an integrated health information system. BMC Health Serv Res. 2008;8:277.

21. Morris JN, Fries BE, Steel K, Ikegami N, Bernabei R, Carpenter Gl, et al. Comprehensive clinical assessment in community setting: applicability of the MDS-HC. J Am Geriatr Soc. 1997;45(8):1017-24.

22. Canadian Institute for Health Information. Continuing care reporting system (CCRS) metadata. Ottawa (ON): The Institute; 2016. (Disponible en français : https://www.cihi.ca/en/types-of-care/ hospital-care/continuing-care/continuing-care-reporting-systemccrs-metadata).

23. Canadian Institute for Health Information. Home care reporting system (HCRS) Metadata: ClHI; 2016.(Disponible en français : https://www.cihi.ca/fr/types-de-soins/soins-communautaires/ services-a-domicile/metadonnees-sisd).

24. Burrows AB, Morris JN, Simon SE, Hirdes JP, Phillips C. Development of a minimum data set-based depression rating scale for use in nursing homes. Age Ageing. 2000;29(2):165-72.

25. Public Health Agency of Canada (PHAC). HIV/AIDS epi updates: national HIV prevalence and incidence estimates for 2011. Ottawa (ON): The Agency; 2014. (Disponible en français : http://www.phacaspc.gc.ca/aids-sida/publication/epi/2010/1-fra.php).

26. Foebel AD, Hirdes JP, Lemick R, Tai JW. Comparing the characteristics of people living with and without HIV in longterm care and home care in Ontario, Canada. AIDS Care. 2015;27(10):1343-53.

27. Stoff DM, Khalsa JH, Monjan A, Portegies P. Introduction: HIV/AIDS and aging. AIDS. 2004;18 Suppl 1:S1-2.

28. Buchanan RJ, Wang S, Huang C. Profiles of nursing home residents with HIV. J Health Care Poor Underserved. 2002;13(3):379-91.

29. Dolder CR, Patterson TL, Jeste DV. HIV, psychosis and aging: past, present and future. AIDS. 2004;18 Suppl 1:S35-42.

30. Justice AC, McGinnis KA, Atkinson JH, Heaton RK, Young C, Sadek $J$, et al. Psychiatric and neurocognitive disorders among HIV-positive and negative veterans in care: Veterans Aging Cohort Five-Site Study. AIDS. 2004;18 Suppl 1:S49-59.

31. Public Health Agency of Canada (PHAC). Population-specific HIV/ AIDS status report: people living with HIV/AIDS. Ottawa (ON): The Agency; 2013. (Disponible en français : http://www.phac-aspc.gc.ca/ aids-sida/publication/ps-pd/people-personnes/index-eng.php).

32. Buchanan RJ, Wang S, Huang C. Analyses of nursing home residents with human immunodeficiency virus and depression using the minimum data set. AIDS Patient Care STDs. 2002;16(9):441-55.

33. Gough K, Karapita S. Facing the future together: An innovative response to the urgent HIV/AIDS crisis in Toronto. Toronto (ON): Casey House, 2011.

34. Counsell SR. 2015 Updated AGS Beers Criteria offer guide for safer medication use among older adults. J Gerontol Nurs. 2015;41(11):60-1.

35. Stevenson DG, Decker SL, Dwyer LL, Huskamp HA, Grabowski DC, Metzger ED, et al. Antipsychotic and benzodiazepine use among nursing home residents: findings from the 2004 National Nursing Home Survey. Am J Geriatr Psychiatry. 2010;18(12):1078-92. 